\title{
WEB-BASED AD-HOC ARCHITECTURE FOR DATA PROCESSING IN A DECENTRALIZED INTERNET
}

\author{
Cătălin BOJA \\ Bucharest University of Economic Studies \\ catalin.boja@ie.ase.ro \\ Cosmin CARTAS \\ Bucharest University of Economic Studies \\ cosmin.cartas@csie.ase.ro \\ Mădălina ZURINI \\ Bucharest University of Economic Studies \\ madalina.zurini@csie.ase.ro
}

\begin{abstract}
Processing large volumes of data is still a performance or a cost issue for many business and also for research, despite current developments in hardware, the increase availability of different Cloud Services or the growing implementation of solutions from different fields like Machine Learning or Artificial Intelligence. The reason is simple, business and clients produce more and more data exceeding too fast the current processing capacity or the cost limits of an owner to process it. In this paper we address the problem of providing data processing capabilities to universities or other institutions that lack a proper infrastructure or required budgets but have access to large volunteer communities that can share their devices and their computation power to form ad-hoc data processing networks for limited time. In order to overcome problems common to the management of large networks and to the distribution of cross-platform software clients we propose a decentralized architecture that will use Internet technologies as the ground base for running Web-based applications.
\end{abstract}

Keywords: Data processing, ad-hoc, Internet, decentralized, Web

JEL classification: L86, C88

DOI: $10.12948 / \mathrm{ie} 2019.06 .14$

\section{Introduction}

For research, processing of large data has become a normal state because the questions for which an answer is desired have become more and more complex and involve solutions from areas like Artificial Intelligence or Machine Learning. Having large volumes of data to train and refine the models is a ubiquitous requirement. Storing these large data volumes and processing them in a limited time are two distinct problems of their own and dealing with them has pushed the IT industry to provide new technologies and services in order to solve them. Some of these solutions have opened new business which provide Cloud services, hardware and also software, on a pay by use approach. But they all rely on the global network, the Internet.

While in a centralized network, a central server is the main target to which users connect along with their data, making data more vulnerable, a decentralization offers a more secure environment in terms of data storage and interaction. Decentralization does not mean just more protection to data, but it also offers more power to the users, giving them complete control over the data they own and share. 
For a system to be considered as being distributed, multiple components having their own behavior must be coordinated using message passing over a network. The components are managed by a single root authority. Adding multiple authorities that control different components, a distributed network can turn into a decentralized one, where no single authority is fully trusted by all others.

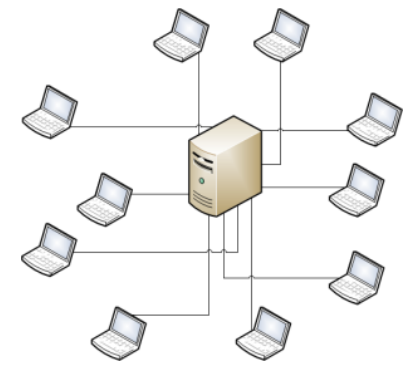

(A) Centralized

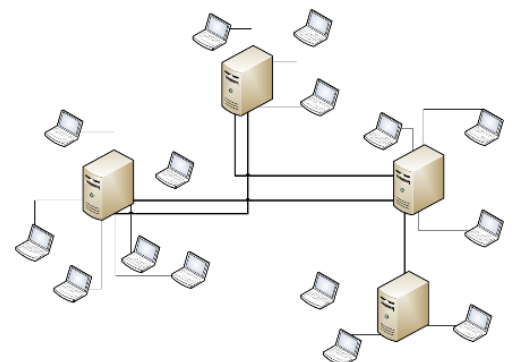

(B) Decentralized

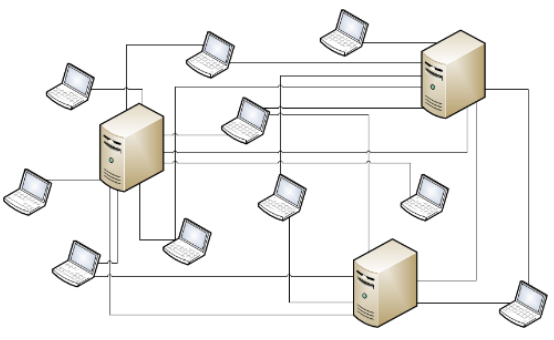

(C) Distributed

Figure 1. Different types of computer systems. Source: authors

Decentralization, as depicted in Figure 1(B), can be obtained by different architectures, such as user-based infrastructure, user-independent infrastructure and hybrid systems. In the case of user-based infrastructure, the networks rely on users for the purpose of resource contribution, bandwidth and storage. For user-independent infrastructure, the system is designed over nodes that are not users. As an example for hybrid systems, we can relate to the case of Tor, where the process is shared also by users and nodes run by third-parties, [1].

The idea of providing decentralized architectures for different Internet services is gaining more momentum as well-known centralized solutions prove to have serious privacy issues, like the Cambridge Analytics scandal [2] or are more prone to major Internet backbone Denial of Service (DoS) attacks [3]. Also, the hype and fast adoption of cryptocurrencies systems, like Bitcoin [4], are supporting the same idea, as they provide a decentralized digital currency systems.

Recently, new decentralized architectures are proposed in order to provide a more user privacy oriented services, like Tim Berners-Lee SOLID project [5], [6], or to provide a more efficient backup and content deliver for Internet available files and resources, [7], [8].

\section{Distributed data processing architecture in a decentralized Internet}

The proposed architecture will address the issue of distributing intensive processing tasks to temporary clients that will share their computing power over limited time periods. The idea is similar to the SETI volunteer distributed computing project [9] in which different users share their computers processing power to analyzed data. This can provide high computational capabilities that can't be achieved with classic investments and budget constraints. For example, a study conducted in 2009 by the BOINCStats platform [10] showed that the clients were providing an average computational power of 617 teraflops to the program. Running the solution implies some costs, it's not free, but they are distributed over multiple clients and it is mostly resumed to some additional costs to the electrical bill.

This kind of volunteer ad-hoc networks offers an efficient intensive data processing solutions to limited budget socio-economic entities. Budget or infrastructure constrains are common problems to universities or public founded research facilities and they need to address it by finding cost efficient and alternative solutions. In this context, the proposed decentralized architecture is designed primarily for environments, like universities, that are described by the existence of:

- a wide network of interconnected devices that combine intranet and Internet connections; 
- a large base of users that use their own devices for computational tasks;

- a community of users that can voluntarily share processor time to others;

- the need to process large volumes of data or to conduct complex experiments that require high computing power.

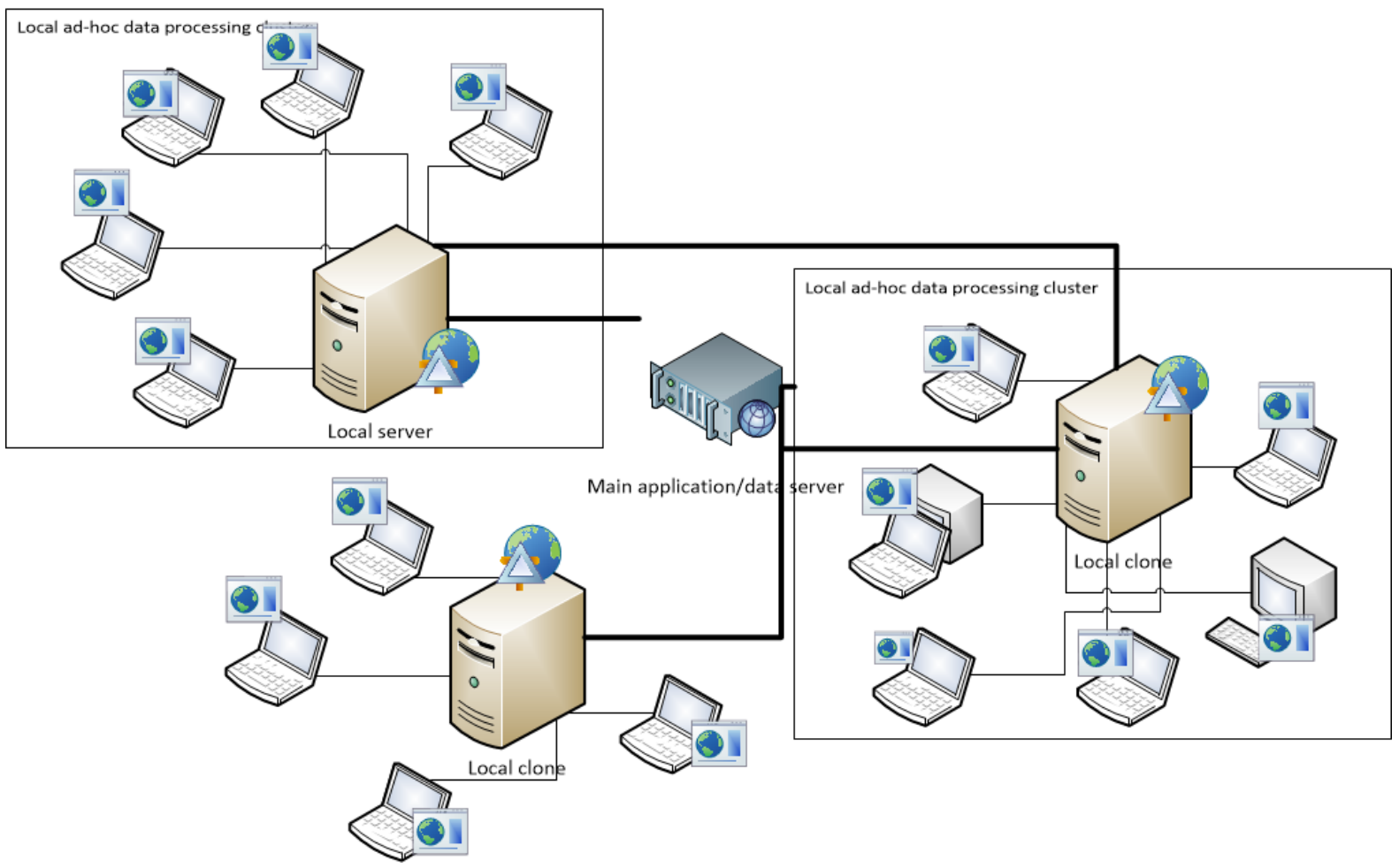

Figure 2. Ad-Hoc architecture for data processing in a decentralized system. Source authors

The proposed architecture, as described in Figure 2, is:

- a decentralized network deployed over Internet or limited to a local intranet; rather than concentrating everything in a single central point; the data is distributed over a multitude of local points that can provide failsafe backup solutions or provide faster access to clients which are in the same network;

- an ad-hoc data processing network as the users will provide their devices capabilities as a volunteer; there are multiple solutions for ad-hoc data processing in the Cloud, like [11], than can be applied in architectures like this one.

- based on Web technologies, as the data processing clients are using a cross-platform solution; the clients are routines that will run in browsers and the communication is conducted over HTTP or HTTPS protocols.

Mapping this concept in a University context is done by

- providing Internet based services that can be accessed from the university own infrastructure or over the Internet; this can be easily implemented as it will reuse the existing infrastructure without changes;

- allowing students and staff to voluntarily share their devices processing capabilities on their own timetable;

- integrating clients devices without the need of additional software as long they have a browser

Moreover, the decentralized approach will assure a high level of privacy as users specific data is stored only on their client device and is available only the users are connected to the network 
and they allow it. Also, common data can be shared between multiple clients acting as a backup system or as a distributed content delivery system.

The proposed architecture needs to address issues regarding:

- synchronizing clients requests for data processing sessions and their response. To minimize the complexity of the communication protocol between nodes and clients we propose a process which is based entirely on client requests in which available clients will have full control over their availability and their request to process data. The architecture is a dynamic one and is controlled entirely by the clients as they will connect to intermediary nodes in order to request data. At any point in time, the number of clients will vary from none to any number of connected clients;

- the distribution of tasks between available clients. Intermediary nodes must synchronize between them in order to manage the consistency of provided data and also in order to distribute available data to clients. For clients this will be a transparent process as they will only request new data and deliver results. In order to reduce complexity a round-robin approach is implemented, as each client will receive next data batch to process when it will request it;

- availability of data and services. Available resources must be efficiently used because in this ad-hoc architecture the system must use at maximum the availability of a large base of clients. Therefore, in this decentralized network overloaded intermediary nodes can redirect client requests to other.

\section{Browser based processing using WebAssembly}

More and more applications are using browsers as the main execution platform. This approach has some significant advantages as

- is providing cross-platform portability because the application will run on any browser, removing any dependencies on the home Operating System; Web applications will provide same user experience on any OS, like Windows, Linux, Chrome OS and others, as long as you can install a browser;

- removes dependencies on hardware and processor types; being able to run an Operating Systems you solve all these dependencies and you decouple them from the Web application development;

- you have a wide range of multiple technologies and frameworks that provide great flexibility in developing different types of Web applications, like Single Page Applications (SPA) [12], Multiple Page Applications (MPA) [13]. Progressive Web Apps (PWA) [14] or just simple plain Web sites; the greater the interest in this area will provide more stable and powerful tools

- less dependencies on other software libraries; the browser will act as an independent execution container for the Web application.

Beside these advantages, users are becoming more mobile and more Web. Usage analysis made by researchers and marketing companies are showing a very big performance boost for solutions that implements web assembly approach. WebAssembly [15], [16] was developed to allow programmers to get access to a set of tools for writing code that runs close to the performance of native implementations. One of the key factor features of WebAssembly is the possibility of parallelizing a task on the web using real multithreading approach. JavaScript is a single threaded sequential language that get use of the browser's event loop that is using web workers. The difference between web workers and threads is that a worker that is used by the browser is not allowed to share mutable data with another worker, thing that is made possible with using threads in WebAssembly [16]. Considering the power of processing found on client devices WebAssembly creates an opportunity that wasn't available before: moving the 
www.conferenceie.ase.ro

execution of heavy operations from server side to client side, resulting in a cost reduction for the servers in cloud. The wasm code that is ran in the browser is executed in a safe memory space, so that gives the frontend world a secure execution context. WebAssembly can be compiled from different languages, such as: $\mathrm{C} / \mathrm{C}++$, Go, Rust or even JavaScript, giving the flexibility of choosing a desired programming language.

\section{Conclusions and further research}

The need for processing large data volumes is becoming a constant for many fields, scientific or socio-economic, and the pace at which new data is collected is much faster than the development of the infrastructure that can store or process that data. Cloud services can fill up this gap but they come with costs, from different perspectives, financial, performance, security and privacy.

One way to address this problem is to try to build that infrastructure and to control it, with the advantages and disadvantages that derive from this approach. The alternative is to build a community in which the effort is distributed among multiple entities which share that control and the costs in order to reach common goals. For distributing data processing this can be easily achieved with today available technologies, both in software and hardware. There are specific contexts in which the data security problems may limit this kind or approach but for public projects or university ones, decentralizing the systems and allowing client to join and share their computational resources may be the natural solution that will overcome budget constraints or limited available infrastructure.

Implementing a prototype of the proposed decentralized architecture will highlight the benefits but also will help identify specific problems which may be not visible from a global perspective, at this moment.

\section{Acknowledgment}

This paper presents results obtained within the PN-III-P1-1.2-PCCDI-2017-0272 ATLAS project ("Hub inovativ pentru tehnologii avansate de securitate cibernetică / Innovative Hub for Advanced Cyber Security Technologies "), financed by UEFISCDI through the PN III "Dezvoltarea sistemului national de cercetare-dezvoltare", PN-III-P1-1.2-PCCDI-2017-1 program.

\section{References}

[1] A. F. Skarmeta, J. L. Hernández-Ramos and M. V. Moreno, “A decentralized approach for security and privacy challenges in the Internet of Things," in 2014 IEEE World Forum on Internet of Things (WF-IoT), Seoul, South Korea, 2014.

[2] T. B. Lee, "Facebook's Cambridge Analytica scandal, explained," March 2018. [Online]. Available: https://arstechnica.com/tech-policy/2018/03/facebooks-cambridgeanalytica-scandal-explained/. [Accessed 1 January 2019].

[3] Cloudflare, "Famous DDoS Attacks," 2019. [Online]. Available: https://www.cloudflare.com/learning/ddos/famous-ddos-attacks/. [Accessed Februar 2019].

[4] N. Satoshi , "Bitcoin: A Peer-to-Peer Electronic Cash System," 31 October 2008. [Online]. Available: https://bitcoin.org/bitcoin.pdf. [Accessed 2019].

[5] T. Berners-Lee, "One Small Step for the Web...," Medium.com, 29 September 2018. [Online]. Available: https://medium.com/@timberners_lee/one-small-step-for-the-web87f92217d085. [Accessed December 2018].

[6] T. Berners-Lee, "SOLID Project," MIT, [Online]. Available: https://solid.mit.edu/. 
www.conferenceie.ase.ro

[7] Protocol Labs, "IPFS (The InterPlanetary File System) Documentation,” 2019.

[Online]. Available: https://docs.ipfs.io/.

[8] C. Famer, "Enabling the distributed web," June 2018. [Online]. Available: https://medium.com/textileio/enabling-the-distributed-web-abf7ab33b638. [Accessed 10 February 2019].

[9] Berkeley SETI Research Center, “SETI@home," UC Berkeley, [Online]. Available: https://setiathome.berkeley.edu/.

[10] W. de Zutter, “SETI@home —Credit Overview,” BOINCstat, December 2009. [Online]. Available: https://web.archive.org/web/20091215135415/http://boincstats.com/stats/project_graph. php?pr=sah. [Accessed January 2019].

[11] D. Logothetis and K. Yocum, "Ad-Hoc Data Processing in the Cloud," in Proceedings of The Vldb Endowment, PVLDB '08, Auckland, New Zealand, 2008.

[12] D. Flanagan, JavaScript - The Definitive Guide, 5th ed, Sebastopol, CA: O'Reilly, 2006.

[13] Neoteric, "Single-page application vs. multiple-page application," 2 December 2016. [Online]. Available: https://medium.com/@ NeotericEU/single-page-application-vsmultiple-page-application-2591588efe58. [Accessed November 2018].

[14] C. Love, "Why Progressive Web Apps Should Be Your Business Choice Over a Native App," 11 March 2019. [Online]. Available: https://love2dev.com/pwa/progressive-webapp-vs-native/. [Accessed March 2019].

[15] W3C Community Group, "WebAssembly Specifications," 2019. [Online]. Available: https://webassembly.github.io/spec/.

[16] A. Haas, A. Rossberg, D. L. Schuff, B. L. Titzer, M. Holman, D. Gohman, L. Wagner, A. Zakai and J. Bastien, "Bringing the web up to speed with WebAssembly," in PLDI 2017 Proceedings of the 38th ACM SIGPLAN Conference on Programming Language Design and Implementation, Barcelona, Spain, 2017. 